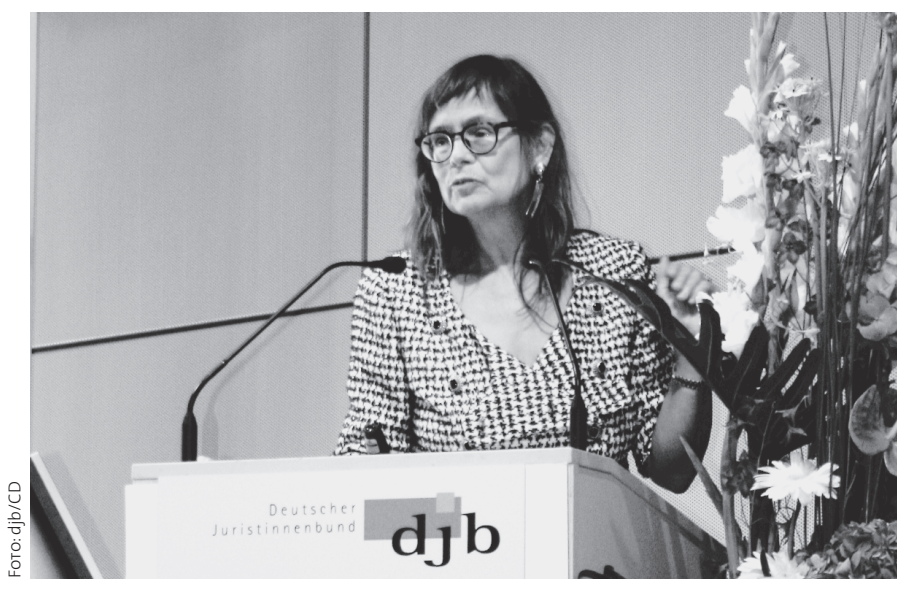

A Mechtild Düsing, Vorstandsmitglied der Arbeitsgemeinschaft Anwältinnen im DAV, Rechtsanwältin und Notarin in Münster, referiert über den Aufstieg im Öffentlichen Dienst und Übertragungsmöglichkeiten auf andere Bereiche aus der Perspektive der Notariate und freien Berufe.

mien seines Fachgebiets) aufzubauen und diese zu pflegen. Geht die Wahl dennoch verloren, so gilt an Sepp Herberger angelehnt: Nach der Wahl ist vor der Wahl!

\section{"Besetzung strategischer Ämter"}

Frauenförderung funktioniert zudem über die Besetzung strategischer Ämter in Institutionen, wie zum Beispiel das der
Genderbeauftragten oder der Vorsitzenden des Genderausschusses. Hier kann Frauenförderpolitik aktiv gestaltet werden. Beispielhaft soll hier der Genderausschuss des DAV genannt werden, dem die Verfasserin vorsteht. Dieser beschäftigt sich künftig verstärkt mit Gesetzesvorhaben, insbesondere mit der Quote für Frauen in Vorständen und Aufsichtsräten.

\section{„Veränderung beginnt in den Strukturen: ausgewählte DAV- Projekte zur Frauenförderung"}

Ohne eine Veränderung der Strukturen, etwa durch die Verankerung von gezielten Frauenförderinstrumenten, wird sich jedoch auch künftig kaum der Frauenanteil unter den Anwältinnen und Notarinnen insgesamt und speziell in den Führungspositionen nachhaltig erhöhen lassen. Der DAV hat vor diesem Hintergrund verschiedene Projekte initiiert, die sich unterschiedlichen Schwerpunkten widmen, jedoch dasselbe Ziel verfolgen: Juristinnen auf ihrem Karriereweg zu unterstützen. Hingewiesen werden soll vor diesem Hintergrund auf die Projekte „Anwältin und Mutter - wie geht das?“, „Anwältinnen in die Aufsichtsräte!", das Mentoring-Projekt der Arbeitsgemeinschaft Anwältinnen im DAV sowie die Verleihung des Anwältinnenpreises. $^{4}$

4 Nähere Informationen zu den Projekten des DAV bietet die Website <www.dav.de> (Zugriff 25.10.2011).

\title{
Der „Praxistest“ beim Zugang zum und Aufstieg im öffentlichen Dienst und Überlegungen für Unternehmen privater Rechtsform
}

\author{
Ursula Matthiessen-Kreuder \\ Personalleiterin, Wiesbaden
}

\section{Einstieg}

Juristinnen haben in der Bundesrepublik der Nachkriegszeit wesentliche Erfolge erzielt bei der Frage, wie sich eine gleichberechtigte Teilhabe von Frauen durchsetzen lassen könnte. Ihre Instrumente waren Gesetzesvorlagen, Gesetzesauslegungen und wissenschaftliche Untersuchungen. Im djb haben sich diese Juristinnen organisiert und ihr Status als Beratungsinstanz und Nichtregierungsorganisation (NGO) steht für die Anerkennung, die sie und ihre Arbeit seit Langem genießen.

Mit der Kampagne „Mehr Frauen in Vorstände und Aufsichtsräte“ hat der djb seit dem Jahr 2009 ein wichtiges Thema aufgegriffen. Es liegt in seiner Kernkompetenz, Regelungen wie den Corporate Governance Kodex aufzugreifen, zu analysieren, frauenspezifische Interessen zu artikulieren und Änderungen und Ergänzungen vorzuschlagen.

Auf dem diesjährigen Kongress hat sich der djb mit der Frage befasst, welche tatsächlichen Folgen die frauenpoliti- schen Bemühungen der letzten Jahre insbesondere im öffentlichen Dienst für den Berufseinstieg gehabt haben.

Versucht man, die tatsächlichen Folgen aus dem Blickwinkel einer Juristin aus einem Unternehmen privater Rechtsform $\mathrm{zu}$ verstehen und zu bewerten, ergeben sich bei der Ausgangslage Gemeinsamkeiten und Unterschiede zum öffentlichen Dienst. Ein gutes juristisches Examen, das Frauen häufiger erreichen als ihre männlichen Kommilitonen, öffnet in angemessenem Verhältnis zu ihren Bewerbungen den Einstieg in den öffentlichen Dienst. Richterinnen und Staatsanwältinnen machen sich auf den Weg und bevölkern die unteren Flure der angesehenen Institutionen. Frauen mit anderen Wünschen wenden sich dem Anwaltsberuf zu und dort stellen sie in der Zwischenzeit eine große Zahl der Kanzleiinhaberinnen. Frauen sind also da.

Juristinnen sind auch da, wenn es in den Unternehmen privater Rechtsform um Einstiegsjobs in der Personalverwaltung geht. Ein Blick auf die Zahlen zeigt, dass Personalverwaltung heute fast ganz weiblich ist, jedenfalls, wenn man die Chefs nicht mitzählt. In der Personalentwicklung sind sie seltener zu 
finden, da hier Bewerberinnen mit anderer Berufsausbildung dominieren. Als Bewerberinnen aber fehlen sie dort, wo Männer typischerweise hinstreben. Frauen mit guten juristischen Examen bewerben sich deutlich seltener oder nie für Traineeprogramme großer Konzerne. Vielleicht wissen sie nicht, welchen breiten Einblick solche Traineeprogramme in Unternehmen ermöglichen. Vielleicht denken sie, dass Juristinnen nicht genommen werden, weil vermeintlich nur betriebswirtschaftliches Fachwissen dominiert. Sie bewerben sich nicht in der IT-Branche, weil sie sich nicht für die Entwicklungen der IT interessieren und dort nie ein Praktikum gemacht haben, und selten in der Chemie, weil sie schon

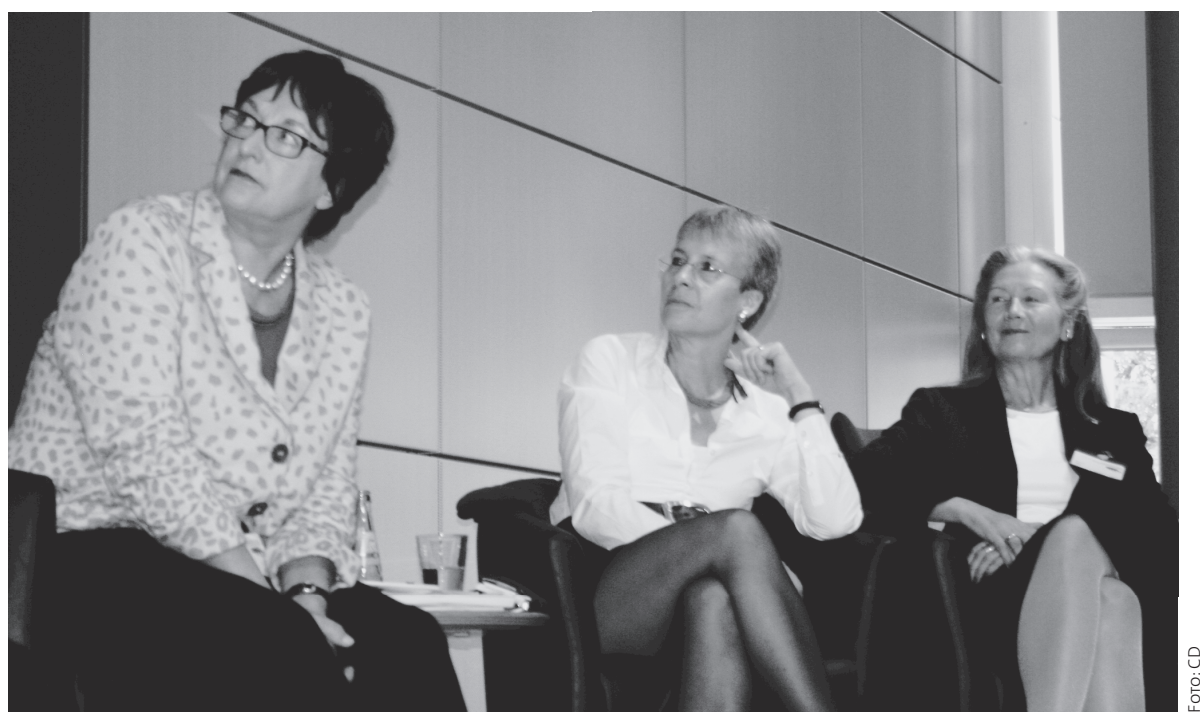

- Es diskutierten Brigitte Zypries MdB (Bundesministerin der Justiz a.D., links im Bild), Ursula Matthiessen-Kreuder (Prokuristin und Personalleiterin) und Ramona Pisal (Vors. Richterin am Oberlandesgericht). im Chemieunterricht in der Schule

schlecht waren. Logistik interessiert sie nur selten und wenn, dann nur aus Sicht der Personalverwaltung. Der Einkaufsbereich eines Unternehmens bleibt ihnen völlig verborgen. Dabei würde sich ein Blick lohnen: Auf das Konzept des angebotenen Traineeprogramms, auf die Ausschreibung der dazu passenden Stellen. Und nachfragen könnten sie: bei Frauen in den anbietenden Unternehmen, bei entsprechenden Berufsverbänden und Studentenvereinigungen. Denn Juristinnen bringen auch für diese Programme eine exzellente Ausbildung, Strukturierungsfähigkeit und Lernkompetenz mit.

Das Problem für die Unternehmen ist ein doppeltes: Nicht nur die mangelnde Bereitschaft gut ausgebildeter Juristinnen, in ihre Branche zu wechseln, schmerzt, sondern auch, dass aufgrund des demografischen Aufbaus dieser Gesellschaft Mädchen und Berufseinsteigerinnen rar werden. Es gibt immer weniger und die gehen als Juristinnen eben lieber in den öffentlichen Dienst.

Bei den Bewerberinnen für die Traineeprogramme oder männerdominierten Branchen steigt der Anteil von nachwachsenden Frauen nur deshalb, weil sich zunehmend Bewerberinnen mit Migrationshintergrund melden. Sie werden sicher dafür sorgen, dass die Unternehmen, für die sie sich entscheiden, moderner und vielfältiger werden. Examinierte Juristinnen orientieren sich eher an den klassischen juristischen Berufen. Vielleicht scheuen sie das vermeintliche Risiko, das sie außerhalb des öffentlichen Dienstes vermuten. Sie trauen sich nicht, jenseits herkömmlicher Ratschläge ihren Weg zu suchen, und sie verkennen, dass ein Berufseinstieg und dann später ein Aufstieg in Branchen, wo Männer viel Geld verdienen können, auch für sie ökonomisch interessant sein kann. Sie denken offensichtlich weniger strategisch und weniger politisch, nicht so sehr in Perspektiven einer Volkswirtschaft und nicht in Entwicklungsmärkten. Auch wenn es ehrenwert ist, sich im juristischen Wertesystem eher herkömmlich zu orientieren, dient aber ein Beruf auch immer der ökonomischen Existenzsicherung, der Frauen selbst und ihrer Familien, kurzfristig und auf
Dauer. Frauen werden im Laufe ihres Berufsleben verstehen, dass Geldverdienen und ökomische Sicherung ihrer Familie wichtige Grundlagen eines selbstbestimmten Lebens sind. Und die meisten von ihnen werden verstehen, dass ökonomische Absicherung auch ein wichtiger Orientierungspunkt für Vereinbarkeitsfragen sein kann.

\section{Aufstieg}

Der djb hat sich auf seinem Kongress 2011 intensiv mit der Frage befasst, ob bestimmte Regelungen Frauen behindern oder wo die Gründe dafür liegen könnten, dass Erfolge frauenfördernder Regelungen hier und da zwar zu verzeichnen sind, aber dennoch manche Berufsebenen im öffentlichen Dienst für Frauen wie vernagelt scheinen. Die veröffentlichten Zahlen entsprechen denen aus Unternehmen der Privatwirtschaft: Je höher man die Hierarchieebenen hinaufklettert, umso weniger Frauen sind Inhaberinnen von relevanten Posten.

Die Bedingungen des Aufstiegs in Unternehmen privater Rechtsform unterscheiden sich deutlich von denen im öffentlichen Dienst: Es gibt seit Jahren weniger begleitendes Regelwerk, das zum Ziel hat, die Organisation mit Gleichstellungsplänen die Dinge analysieren und Veränderungsmaßnahmen festlegen zu lassen. Gleichstellungsstellen und Frauenbeauftragte gab es in Unternehmen privater Rechtsform hier und da, gibt es weiterhin hier und da, aber es fehlt ihnen die Kraft und Akzeptanz, wirkliche Veränderungen zu erreichen. Denn sie stehen außen, außerhalb der Machtstrukturen erfolgreichen Wirtschaftens. Und Unternehmen entwickeln sich, das weiß jeder Mann, rund um das erfolgreiche Geschäftemachen. Da, wo das Geld verdient wird, sind Innovation und Veränderung zu Hause. Dort müssen Frauen ansetzen, wenn sie mitreden und gestalten wollen. Externe Regelungen helfen nur eingeschränkt weiter.

Das bedeutet, dass Frauen und auch Juristinnen anders über Berufsaufstieg nachdenken müssen, wenn sie sich in einem Unternehmen privater Rechtsform zurechtfinden müssen: Wenn 
sie in gutem fachlichen Ehrgeiz meinen, dass ihre Fachlichkeit mit großer Wahrscheinlichkeit nach oben führen wird, dann irren sie sich. Wenn sie andererseits das Thema Führen für sich entdeckt haben, weil sie meinen, dass dies Frauen natürlicherweise mehr und auch ihnen liegt, verkennen sie, dass Führen in keiner Organisation ein Selbstzweck ist, sondern sich immer daran orientieren muss, was das Unternehmen oder die Organisation gemeinsam erreichen will.

Aber es gibt Rezepte, über die es sich für Juristinnen nachzudenken lohnt: Der Aufstieg in die nächste Hierarchieebene gelingt Frauen in Unternehmen privater Rechtsform häufig nur, wenn sie das Unternehmen wechseln. Leider ist die „Gläserne Decke“ überall. Wenn es nicht weitergeht, weil Chefs sich nicht bewegen oder ihnen ähnliche, weil männliche Konkurrenten vorgezogen werden, dann sollte „frau“ gehen. Die Chancen stehen heute gut, dass ein Quereinstieg in ein anderes Unternehmen gelingt, denn viele Unternehmen sehen sich starkem Druck ausgesetzt, Frauen für die nächste Hierarchieebene wenigstens von außen zu rekrutieren.

Internationale Einsätze sind für viele Männer heute der wichtigste Baustein einer wirklich großen Karriere. Frauen gehen dann meist als Partnerinnen mit. Warum die Dinge partnerschaftlich nicht anders denken: Sie geht für ihr Unternehmen ins Ausland, in der Einkaufsabteilung zum Beispiel, die sie inzwischen für sich entdeckt hat. Sie regelt diesen Einschnitt mit Partner und Kindern so, wie er das bisher auch getan hat. Es geht, bringt Freude und der ganzen Familie unter Umständen eine erhebliche Erweiterung ihres Horizonts.

Aufstiegskiller Nr. 1 ist die Vereinbarkeit von Beruf und Familie, so heißt es. Diese These wird sich auf Dauer gegen die Frauen wenden. Zum einen ist die Vereinbarkeit in der Regel für den echten Aufstieg in eine relevante Position nicht mehr brennend, da Frauen in diesem Alter üblicherweise mit den passenden Männern ihre Familiengründungsphase abgeschlossen haben. Auch hat jede aufstiegswillige Frau dieses Thema im Griff. Unternehmen und Organisation werden nur dann auf die einseitige Zuordnung dieses Themas zu Frauen verzichten, wenn Vereinbarkeit auch von Männern eingefordert wird. Arbeitgeber werden trotz vorübergehender Familienphase Karrieren nur dann auch für Frauen zufriedenstellend ermöglichen, wenn Frauen und Männer sie gleichermaßen einfordern und beide berufliche Flexibilität für diesen kurzen Zeitraum einer lebenslangen Karriere verlangen.

Netzwerke sind ein Phänomen, was Männer regelmäßig zum Aufstieg verhilft. Man kennt sich, man vertraut sich, man holt sich und man zählt aufeinander, wenn es darum geht, die beruflichen Dinge zur Zufriedenheit der Männer zu regeln. Frauen haben Netzwerken für sich als Thema erkannt und besprechen es, wo immer sich dafür eine Plattform findet. Aber sie handeln häufig nicht unterstützend, vermittelnd, miteinander netzwerkend.

Mentoring ist ein Instrument, das jede Frau nutzen sollte. Was spricht dagegen, sich im Internet Angebot für unternehmensübergreifende Mentoringprogramme zu suchen? Was spricht dagegen, den eigenen Verband aktiv nach einer passenden Mentorin abzusuchen? Mentoring ist das einzige Instrument, wo männliche Kollegen keinen Startvorteil haben.

Headhunter verkünden regelmäßig in den Tageszeitungen, wie sehr sie sich die Vermittlung kompetenter Frauen wünschen. Auch wenn es leider so ist, dass viele Headhunter keine praktischen Vorschläge für viele suchende Frauen haben, so sollten sich aufstiegswillige Frauen unbedingt bei Headhuntern ins Gespräch bringen und sie von ihrem Potential überzeugen. Sie können Unternehmenswechsel ermöglichen oder eine interne Aufstiegsperspektive eröffnen, wenn „frau“ mit einem attraktiven Arbeitsvertragsangebot des Wettbewerbers vorspricht.

Auf den ersten Blick scheint das Thema „Rekrutierung“ nicht in den Strauß möglicher aufstiegsfördernder Maßnahmen zu passen. Aber in Unternehmen der privaten Rechtsform, wo Hierarchieebene nicht in Stein gemeißelt sind und zunehmend Projektarbeit mit eigenen Projekthierarchien dominiert, können gezielte Neueinstellungen auch Frauen nützen. Nachwachsende Mitarbeiterinnen oder Mitstreiterinnen gleicher Ebene einzustellen, kann ein sehr kluger Schachzug sein, freundschaftliche Bande in Unternehmen wirksam zu machen.

Der djb wird sich weiterhin sehr stark für die Erhöhung des Anteils von Frauen auf Seiten der Anteilseigner in Aufsichtsräten stark machen. Auch die Übernahme von solchen Mandaten ist für Frauen Ausweis und Bestätigung ihres Aufstiegswillens und ihrer Aufstiegsfähigkeit und sollte in seiner Bedeutung für Karriere nicht unterschätzt werden.

\section{Mittelbare Entgeltdiskriminierung in Tarifverträgen des öffentlichen Dienstes - ein Ende in Sicht?}

\section{Zugleich ein Beitrag zum Verständnis und zur Prüfung von Entgelt(un)gleichheit}

\author{
Prof. Dr. Gertraude Krell \\ Universitätsprofessorin a.D., Berlin \\ Dr. Karin Tondorf \\ Wissenschaftlerin und Beraterin, Seddiner See
}

\begin{abstract}
„Diskriminierungsfreie(re) Entgeltgestaltung - leider immer noch ein Prinzip ohne Praxis“, konstatierte kürzlich Regine Winter. ${ }^{1}$ Dass das auch für den öffentlichen Dienst gilt, zeigen wir im Folgenden - und setzen den Schwerpunkt auf mittelbare Entgeltdiskriminierung.
\end{abstract}

